In Crescendo. Institucional. 2015; 6(1): 133-145

\title{
Planeación a largo Plazo de una EMPRESA de tRANSPORTE TERRESTRE DE PASAJEROS: MODELO TEÓRICO
}

\section{LONG-TERM PLANNING OF A PUBLIC LAND TRANSPORT COMPANY: THEORETICAL MODEL}

\author{
Oswaldo Robles Gutiérrez*
}

\begin{abstract}
RESUMEN
$E^{1}$ propósito del presente artículo de investigación es explicar cómo debe funcionar una empresa de transporte terrestre de pasajeros, encomiendas y traslado de dinero, que utiliza cómo técnica la planeación a largo plazo. Pero ¿qué es la planeación a largo plazo y por qué es importante? Brevemente diremos que mantiene el estudio empresarial tanto en el futuro como en el presente, refuerza los principios adquiridos en la misión, visión y estrategia, fomenta la planeación y la comunicación interdisciplinarias, asigna prioridades en el destino de los recursos, es el puente con el proceso de planeación táctica que obliga a los ejecutivos a ver la planeación desde las perspectivas del presente en responsabilidades de cumplimiento de metas futuras, mantiene unido al equipo administrativo para traducir la misión, visión y estrategia en resultados tangibles.

La planeación a largo plazo lleva a la posición, proporciona las herramientas para establecer un enfoque de las posiciones que la empresa requiere alcanzar, como mercados futuros, tecnología, desarrollo humano y previsiones financieras.

La empresa que se estudió tiene tres actividades fundamentales: transporta pasajeros, ofrece servicios de transferencia de dinero y presta servicios de carga y encomiendas. Actualmente opera en las siguientes rutas: parte desde la ciudad de Lima hacia el norte,
\end{abstract}

* Economista. administrador general de la Empresa Internacional de Transportes Turísticos y Servicios SRLtda. ITTSA. 
y llega a Chimbote, Trujillo, Chiclayo y Piura, y desde estas ciudades, a Lima; de Chimbote a Piura, Paita y viceversa, y de la ciudad de Trujillo a Piura, Sullana, Paita, Talara y viceversa.

El objetivo básico de la investigación es elaborar la planeación a largo plazo de una empresa de transporte de pasajeros, encomiendas y traslado de dinero. La planeación a largo plazo de las actividades futuras de la empresa, en los próximos cinco años, permitirá elaborar el plan a largo plazo de producción, el plan a largo plazo de recursos humanos, el plan a largo plazo de flujo de fondos, el plan a largo plazo de impuestos, el plan a largo plazo de marketing y el plan a largo plazo de servicio al cliente.

Palabras clave: planeación a largo plazo, gestión, posición, rentabilidad.

\begin{abstract}
The purpose of the present research is to explain how long-term planning must operate in a company of public, package and money land transport. But what is the long-term planning and why it is important? It maintains the business both in the future and the present, reinforces the principles acquired in the mission, vision and strategy, promotes interdisciplinary planning and communication, assigns priorities on the destination of the resources, it is the bridge with the planning process tactics required by executives to see the planning from the perspective of the present responsibilities to fulfill the goals of the future, holds together the administrative team to transform the mission, vision and strategy into tangible results. Long-term planning leads to the position, provides the tools to establish an approach.

The studied company has three main activities: transport of people, money and packages. Currently operates on the following routes: it departs from the city of Lima to the North, arriving to the cities of Chimbote, Trujillo, Chiclayo and Piura; then, from these cities, back to Lima; from Chimbote to Piura, Paita and vice versa; and from Trujillo to Piura, Sullana, Paita, Talara, and vice versa. The main goal of this research is to develop a longterm planning for a people, money and packages land transport company. The long-term planning of the future activities of the company in the next five years will allow to develop the long-term plan of production, long-term plan of human resources, long-term plan of flow of funds, long-term plan of taxes, long-term plan of marketing, and long-term plan of customer service.
\end{abstract}

Keywords: long-term planning, management, position, profitability. 


\section{INTRODUCCIÓN}

La filosofía de planeación a largo plazo de una empresa de transporte de pasajeros, encomiendas y traslado de dinero, incluye la aplicación de la intuición de la experiencia empresarial, hecho que permitirá determinar las posiciones futuras de la empresa, motivo de nuestra investigación.

La planificación a largo plazo de la empresa en mención permitirá establecer la estrategia que nos conducirá a:

1) Mantener a la empresa en su presente y futuro.

2) Reforzar los principios adquiridos en la misión, visión y estrategia de la firma.

3) Fomentar constantemente la comunicación entre los ejecutivos y los trabajadores de la empresa.

4) Asignar prioridades en los destinos de los recursos de la empresa.

5) Ser el puente con el proceso de planeación táctica.

6) Comprometer a los ejecutivos para que vean la planeación desde las perspectivas del presente como responsabilidades del cumplimiento de las metas futuras.

7) Mantener al equipo administrativo para traducir la misión, visión y estrategia en resultados tangibles.

La presente investigación se realizó durante el período 2014 para su aplicación en el período 2015-2019, el que se desarrollará dentro de la teoría de la planificación estratégica de las organizaciones privadas, fundamentalmente: la planeación a largo plazo de una empresa de transporte de pasajeros, encomiendas y traslado de dinero. Todo esto nos lleva a plantearnos la pregunta: ¿cómo elaborar y aplicar un plan a largo plazo para mejorar la posición y rentabilidad en la empresa de transporte terrestre de pasajeros, encomiendas y traslado de dinero en el período 2015-2019?

Planteado el problema, surgen las siguientes interrogantes: ¿por qué debe elaborarse un plan a largo plazo?, ¿qué elementos debe contener el plan a largo plazo?, ¿cómo deben relacionarse los elementos del plan a largo plazo?, ¿cómo debemos evaluar los resultados del plan a largo plazo? y ¿cuáles son los beneficios del plan a largo plazo? Todo ello nos permite plantear los siguientes objetivos: 


\section{Objetivo general}

Elaborar y aplicar un plan a largo plazo para mejorar la posición y rentabilidad en la empresa de transporte terrestre de pasajeros, encomiendas y traslado de dinero durante el período: 2015-2019.

\section{Objetivos específicos}

- Determinar los elementos básicos que debe contener el plan a largo plazo.

- Relacionar los elementos del plan a largo plazo.

- Evaluar los resultados del plan a largo plazo.

- Determinar la rentabilidad y los beneficios anuales del plan a largo plazo y mejorar la posición de la empresa.

\section{Hipótesis}

La aplicación de un plan a largo plazo en una empresa de transporte terrestre de pasajeros, encomiendas y traslado de dinero para el período 2015-2019 nos permite mejorar la rentabilidad económica y el posicionamiento en el mercado del sector transporte.

La presente investigación es de carácter práctico, tiene como finalidad implementar en una empresa de transporte terrestre de pasajeros, encomiendas y traslado de dinero las técnicas empresariales de planeación a largo plazo. Este hecho va a contribuir a mejorar la posición y rentabilidad de la empresa de transportes, generadora de empleo y de buen servicio ofrecido en sus diferentes concesiones a los usuarios de la empresa.

La viabilidad de la investigación se está ejecutando con la disponibilidad presupuestaria personal, con la recolección de datos informativos a nuestro alcance, con la capacidad metodológica dada por la Escuela de Posgrado de la UNT y, sobre todo, con el apoyo de los directivos de la mencionada empresa.

Las consecuencias de la investigación van a permitir mejorar la posición de la empresa en el sector transportes; incrementar los ingresos y beneficios de la empresa en estudio; ampliar el servicio de pasajeros, giros y encomiendas con nuevas concesiones; mejorar el servicio de pasajeros en los diferentes puntos de las rutas concesionadas; seguridad de viaje de los pasajeros y mejora en la entrega de giros y encomiendas; incrementar las tasas de empleo, la rentabilidad de la empresa y los aportes tributarios.

Esta investigación es de carácter explicativo porque "va más allá de la simple descripción de conceptos o de fenómenos, o del establecimiento de relaciones entre conceptos" (Hernández, 2006: 108-110). La investigación se centra en explicar por qué ocurre un fenómeno y en qué condiciones se da, o simplemente por qué dos o más variables están interrelacionadas.

El marco referencial de esta investigación comprende: 


\section{Marco teórico}

La planeación a largo plazo incluye ideas y aplicaciones de intuiciones gerenciales para determinar las posiciones futuras de una organización. Pero entiéndase la expresión no en sentido estático, sino dinámico y flexible, capaz de: a) mantener una relación permanente entre el presente y el futuro; b) permitir mirar y revisar constantemente los principios de la misión, visión y estrategia de la empresa; c) asignar prioridades en el destino de los recursos para la planeación táctica; y d) comprometer a los ejecutivos para que vean la planeación desde las perspectivas del presente como responsabilidades del cumplimiento de las metas futuras.

Dentro de este marco, hemos tratado de elaborar un modelo de planeación a largo plazo que responda, entre el juicio teórico de la planeación estratégica y la vida real de la empresa de transporte de pasajeros. Este modelo teórico lo formulamos a continuación.

\section{MODELO DE PLANEACIÓN ESTRATÉGICA}
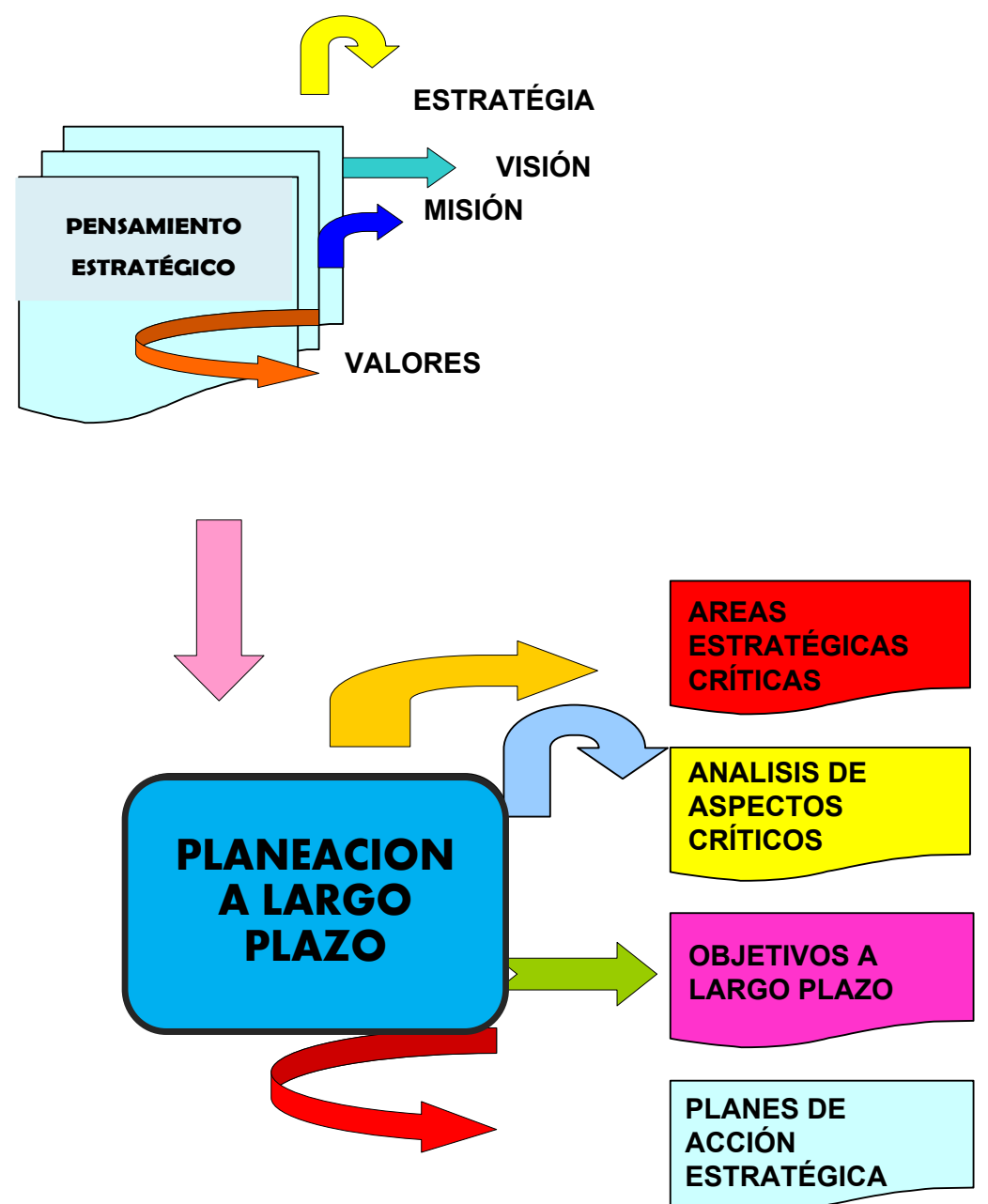

El diagrama de la planeación estratégica explica por sí solo el marco teórico de nuestra investigación. 


\section{Marco conceptual}

Algunos conceptos usados en nuestra investigación, como:

\section{Visión}

Se refiere a una mirada hacia el futuro, esto es, cómo deseamos que la empresa o negocio sea en el futuro. Cómo deseamos que nuestros clientes y otros nos vean en lo por venir. Que la visión de la empresa que buscamos analizar permita encontrar una respuesta suficientemente clara a las siguientes preguntas:

- ¿Qué productos o servicios estamos ofreciendo en el mercado?, ¿son rentables?, ¿en qué medida?

- ¿Por qué debemos pensar que nuestros productos deben ser diferentes de los de la competencia?

- ¿Qué beneficios van a traer estos productos o servicios a los clientes?, ¿cuál es nuestro beneficio?

- ¿Cómo vamos a estructurar nuestras relaciones con los proveedores y qué esperamos de ellos?

- ¿Cómo vamos a organizar la distribución de los productos o servicios?

- ¿Cómo pretendemos desarrollar nuestra propuesta de valor para los empleados?

- ¿Por qué debería trabajar nuestra masa laboral para nosotros?

- ¿Qué nuevos productos pueden ofrecerse en el mercado?, ¿son suficientemente rentables?

\section{Valores estratégicos:}

Hay un gran número de ideas que pueden considerarse como valores estratégicos. Para desarrollar un plan estratégico es recomendable establecer un conjunto de siete valores estratégicos orientados a la empresa objeto de nuestro estudio y que a continuación definiremos:

- Ética: La empresa de transportes debe atraer a los clientes hacia los valores éticos. Por ejemplo, si el pasajero hace una protesta fuerte, el trabajador de la empresa debe tener la delicadeza de conducir su respuesta en forma fina y adecuada. No olvidar la máxima: el cliente siempre tiene la razón.

- Calidad: Considerada como valor, asegúrese de que la respuesta del trabajador se acompañe de una acción apropiada y que satisfaga al cliente dentro del círculo de la eficiencia.

- Seguridad: Las unidades de transporte son cada día más atractivas para la delincuencia. La empresa debe implementar todas las herramientas de seguridad durante la trayectoria del vehículo ante posibles asaltos. 
- Innovación: Cuando se toma como valor este término, se debe equilibrar entre la innovación y el costo. Hilvanar con sumo cuidado este concepto, ya que es un valor que fuertemente acompaña a la competencia.

- Imagen: La imagen institucional es un valor fuertemente competitivo en el mercado de transporte de pasajeros y encomiendas.

- Responsabilidad con los clientes: Deben ser atendidos por empleados con fuerte carisma personal. Deben tener en cuenta que el trato con clientes es diferente de cualquier otro.

- $\quad$ Rentabilidad: Se debe entender que este es un valor fuertemente necesario para la vida de la empresa. Además, se pueden permitir pérdidas del costo fijo en el corto plazo, pero no en el largo plazo, puesto que la esperanza actualizada de la utilidad debe ser positiva en el largo plazo, de lo contrario se debe abandonar el negocio y buscar en qué sector de la economía pueden los empresarios ser más eficientes para el manejo gerencial.

\section{Misión}

Es una declaración duradera sobre el propósito que distingue a una empresa de otra similar. Es la declaración de la "razón de ser" de una empresa. Responde a la pregunta clave "¿cuál es nuestro negocio?". Para tener una declaración clara y precisa de la razón de ser de una empresa, debe estar contenida y definida en forma exacta en la misión, y de este modo poder establecer objetivos y formular las estrategias que encaminarán a la visión. Es recomendable que la declaración de la misión responda por lo menos a las siguientes preguntas:

- ¿Cuál es nuestro negocio?

- ¿A quién servimos?

- ¿Cuáles son nuestros principios?

\section{Estrategia}

El significado del término "estrategia", proviene de la palabra griega strategos, que significa 'jefe de un ejército'; tradicionalmente utilizada en el terreno de las operaciones guerreras.

En los últimos años el concepto de estrategia ha evolucionado de manera tal que, sobre la base de este concepto ha surgido una nueva escuela de administración y una nueva forma de dirigir a las organizaciones que hoy llamamos "administración estratégica".

El empleo del término "estrategia" en administración es mucho más rico que las acepciones militares; la estrategia en administración se define como la ciencia y el arte de administrar cosas con el uso del talento humano en las diversas actividades del hombre. Mientras que, en las fuerzas armadas, es el acto belicoso de los hombres que pretenden 
dominar a la fuerza a otros hombres, dentro y fuera de una nación, para conseguir fines no democráticos.

\section{Posicionamiento}

La estrategia debe elegir el posicionamiento requerido por la empresa y sus productos o servicios en la mente del consumidor. Podemos decir que posicionamiento es encontrar un espacio vacío en la mente del cliente, ocupándolo antes de que lo haga la competencia. Esta acción es el resultado de dos maniobras estratégicas: la segmentación y la diferenciación.

En teoría de mercados, posicionar es recortar segmentos del mismo mercado, por ejemplo, cuando se necesiten o deseen cosas distintas de otros segmentos y construir un producto para adueñarse de esa diferencia. En términos más simples, es hallar el "nicho" que conduce a la "eternidad de los negocios". Por eso debemos afirmar que el posicionamiento no se refiere al producto o servicio, sino a lo que se hace con la mente de los probables clientes o personas sobre quienes se desea influir.

El posicionamiento es esencialmente una estrategia de negocios, responde a la pregunta: ¿cómo diferenciarse de los demás? Una vez que la gerencia sabe quién es y qué la diferencia del otro u otros, todas las decisiones se facilitan. Además, existen posicionamientos basados en la edad o sexo de los clientes, como por ejemplo, productos como Billiken o Aspirinetas, dirigidos al público infantil.

Otros posicionamientos en la mente de los consumidores son los diferentes tipos de marcas desarrolladas en el mercado. Asimismo, aquellas que se desarrollan en forma casi natural, como las estrategias de extensión de líneas y de marcas; por ejemplo, las refinerías de maíz que permiten construir nuevos negocios a partir del tradicional posicionamiento saludable del aceite Mazola, etc. Cualquier producto, en este caso el aceite de maíz, puede llevar como estrategia el posicionamiento.

La conclusión acerca del posicionamiento es que jamás un producto tiene un solo posicionamiento, y la pregunta de referencia puede ser: ¿cuál de los posibles posicionamientos resulta más valioso, en un momento dado, para fijar mejor en la mente el producto en referencia? He aquí algunas respuestas:

- Que el producto o servicio esté bien posicionado en sus respectivos segmentos.

- Que el producto no tenga ningún posicionamiento, que no se sepa quién ni por qué lo consume.

- Que el producto tenga un mal posicionamiento.

- Que el producto tenga un posicionamiento indefinido. Por ejemplo: el helado.

- Que el producto pueda quedar desposicionado. Por ejemplo: productos para la salud desposicionan al cigarrillo, a las bebidas alcohólicas y a las grasas. 
El éxito de la investigación se alcanzará cuando se cumpla la misión, visión y estrategia de la empresa de transporte de pasajeros y esto exige resolver las áreas de resultado crítico indicadas en el siguiente cuadro:

\begin{tabular}{|c|c|}
\hline \multicolumn{2}{|c|}{ ÁREAS DE RESULTADOS DE LA GESTIÓN ESTRATÉGICA DE LA EMPRESA } \\
\hline OBJETIVOS ESPECÍFICOS & POLÍTICAS O ACCIONES \\
\hline $\begin{array}{l}\text { 1. Ingresos y ventas } \\
\text { 2. Ingresos netos }>\text { costos + impuestos } \\
\text { 3. Ampliación de mercados } \\
\text { 4. Adquisiciones y renovación } \\
\text { 5. Satisfacción al cliente } \\
\text { 6. Servicios al cliente } \\
\text { 7. Resultados operativos }\end{array}$ & $\begin{array}{l}\text { Desempeño de la línea de servicios } \\
\text { Margen positivo de resultados } \\
\text { Marketing estratégico } \\
\text { Adquisición de nuevos buses } \\
\text { Calidad y seguridad de servicio } \\
\text { Elaboración de un código de ética } \\
\text { Evaluación de estados financieros }\end{array}$ \\
\hline OBJETIVO GENERAL & POLÍTICAS \\
\hline $\begin{array}{l}\text { Alcanzar una tasa interna de retorno de largo } \\
\text { plazo (i) más alta que la tasa de mercado. }\end{array}$ & $\begin{array}{l}\text { - Inversiones selectivas en buses } \\
\text { - Invertir en desarrollo y tecnología } \\
\text { - Inversiones en compra de nuevos locales } \\
\text { - } \text { Mejorar la calidad de los servicios } \\
\text { - Aplicación de nuevas técnicas de gestión } \\
\text { Aplicación del marketing de transporte }\end{array}$ \\
\hline
\end{tabular}

A partir de las ideas de los modelos de planeación a largo plazo y de las políticas o acciones que permitan elaborar y aplicar un plan a largo plazo para la empresa de transporte de pasajeros durante el período 2015 - 2019, la pregunta será: ¿cómo es que la aplicación de este plan a largo plazo permitirá mejorar la posición y rentabilidad de la empresa de transporte de pasajeros, encomiendas y traslado de dinero?, teniendo como variable dependiente la posición y rentabilidad de la empresa, mientras que la variable independiente será la aplicación del plan a largo plazo.

Estos planes a largo plazo deben contener, fundamentalmente:

\section{a) Áreas estratégicas críticas}

Estas son las principales categorías hacia las cuales debe enfocarse la atención para el futuro previsible. El enfoque de las áreas estratégicas críticas es de alcance amplio y diseñado para la determinación de dónde se quiere estar como organización, y no tanto de los resultados específicos que se quieran alcanzar, así tenemos que al enfocar la proyección financiera como áreas estratégicas críticas, ello tiene que ver con tasas de crecimiento, valor neto y combinación de utilidades. Enfocar desarrollo de productos como un área estratégica crítica consideraría a nuevos productos o servicios de largo desarrollo con un buen potencial a futuro. El principal propósito de determinar las áreas estratégicas críticas es ayudar en la identificación de los aspectos críticos estratégicos que necesitan ser abordados durante un amplio período para cumplir con la misión y la estrategia. 


\section{b) Análisis de aspectos críticos}

Aborda oportunidades y amenazas externas, fortalezas y limitaciones internas que tendrán efecto en el cumplimiento de la misión y la estrategia, que requerirán de más de un año para resolverse; así tendremos que un aspecto estratégico crítico sería la obsolescencia proyectada de los productos o servicios de alto volumen y alto margen. Los aspectos estratégicos críticos tienden a estar más orientados hacia la oportunidad.

\section{c) Objetivos a largo plazo}

Representan las posiciones estratégicas que se desean alcanzar en un momento dado del futuro. Este enfoque de la planeación a largo plazo incluye proyecciones financieras como parte de los objetivos a largo plazo y no como un elemento separado. Todos los objetivos a largo plazo tienen implicaciones financieras, y todas las proyecciones financieras dependen de otros objetivos a largo plazo.

Los objetivos a largo plazo pueden establecerse sin necesidad de saber cómo alcanzarlos; es válido establecer una posición en el horizonte para lograr una buena contribución a la misión y a la estrategia, y luego proveer los medios de los que se valdrá.

\section{d) Planes estratégicos de acción}

Identifican los pasos o puntos de referencia que se requieren para avanzar hacia los objetivos a largo plazo. Los planes estratégicos de acción serán multianuales y multifuncionales; algunas partes tendrán lugar durante dos o más años y requerirán de esfuerzos de más de un área en función, como marketing, investigación y desarrollo, producción, recursos humanos, etc.

Los planes estratégicos de acción son los puentes entre los planes tácticos y los de largo plazo para el logro de las metas generales.

En consecuencia, con el desarrollo del presente trabajo de investigación se conseguirán resultados en el largo plazo necesarios para que la empresa brinde calidad, eficiencia y seguridad en el servicio de transporte terrestre de pasajeros, así como en el traslado de encomiendas, giros y valores, a través de una atención personalizada que garantice la satisfacción de los clientes y ser la empresa líder en el sector, lo que redundará en una mejor posición de la empresa en el rubro transporte de pasajeros, un incremento de la rentabilidad y generación de empleo.

\section{MATERIAL Y MÉTODOS}

Población: Para el presente estudio, comprende el total de la población laboral de la Empresa Terrestre de Pasajeros Internacional Transporte Turístico y Servicios SRLtda. ITTSA, así como todos los ejecutivos y trabajadores de cualquier nivel. 
Muestra: La misma que la población.

Métodos y técnicas de investigación: El método que se utilizó para contrastar la hipótesis fue la investigación de tipo explicativo, y la diagramación para explicar el proceso de investigación del plan a largo plazo responderá al siguiente modelo de investigación.

\section{MODELO DE INVESTIGACIÓN:}


\section{IMPLEMENTACIÓN \\ DE LA ESTRATEGIA}

SISTEMA DE

VALORES

Las técnicas de investigación que se utilizaron fueron la técnica documental y técnica de campo. La técnica documental comprendió la recopilación de información para enunciar las teorías que sustentan el estudio a desarrollar; la técnica de campo consiste en entrevistas y sondeos de opinión que nos permitirán confrontar la teoría con la práctica. 


\section{RESULTADOS}

Resultados de la planeación a largo plazo de la Empresa Terrestre de Pasajeros Internacional Transporte Turístico y Servicios SRLtda. ITTSA Giros y Encomiendas:

- $\quad$ Ingreso neto, costos más impuestos

- Ampliación de mercados: nuevas concesiones

- Adquisiciones y renovaciones de ómnibus

- Resultados operativos

- Tasa interna de retorno a largo plazo

- Aumentar la satisfacción del cliente con nuestros servicios y nuestros colaboradores

\section{DISCUSIÓN}

La competencia de mercado en las empresas de transporte de pasajeros, encomiendas y giros de dinero siempre ha sido muy dura. Por eso, podemos afirmar que la empresa ha operado en un mercado muy competitivo; algo así como los mercados perfectos, nombre muy conocido en la teoría microeconómica. En ellos, cada empresa busca sobresalir para captar la mayor cantidad de clientes. Además, le gustaría operar en un mercado monopólico, donde las utilidades son absolutas. A veces, las empresas de transporte han tenido que trajinar como si subiesen las laderas de un cerro: demasiado empinadas y duras. Mas la experiencia de nuestros ejecutivos ha sido no solo buena, sino muy buena y eficiente. Las empresas de transporte terrestre de pasajeros siempre han trajinado por el camino de la competencia perfecta.

\section{CONCLUSIONES}

1. La empresa de transporte de pasajeros bajo nuestro estudio y análisis ha operado siempre en un mercado competitivo, lo que en teoría suele llamarse competencia perfecta.

2. Se observa que la técnica de planeación a largo plazo es un buen instrumento para analizar el comportamiento futuro de las empresas de transporte de pasajeros del tipo que aquí analizamos.

3. El éxito de las empresas de transporte de pasajeros es, en gran medida, consecuencia de la habilidad gerencial de sus ejecutivos. 


\section{REFERENCIAS BIBLIOGRÁFICAS}

Bueno Campos, Eduardo. Organización de empresas: estructura, procesos y modelos. Ediciones Pirámide, 2. a edición, Madrid - España, 1997.

Bunge, Mario (2002). Crisis y reconstrucción de la Filosofía. Gedisa, 1. a edición.

Díez C., José, A. Ulises Moulines (1997). Fundamentos de Filosofía de la Ciencia. Ed. Ariel SA, Barcelona.

Florida, Marcial (2003). Epistemología (enfoque reconstructivo). Universidad de Cajamarca - Facultad de Educación, Cajamarca.

Goodstein, Leonard, Nolan, Timothy M. y Pfeiffer, J. William. (2000). Planeación estratégica aplicada. McGraw-Hill, 1. ${ }^{a}$ ed., Colombia.

Hernández, Roberto, Fernández-Collado, Carlos y Baptista, Pilar. (2006). Metodología de la investigación. México: McGraw- Hill, 4. ${ }^{a}$ ed.

Hill W.L., Charles. Administración estratégica. (2004). México: McGraw-Hill, 6. a ed.

Kuhn, Thomas (1996). ¿Qué son las revoluciones científicas? Barcelona: Paidós, ICE Universidad Autónoma de Barcelona. (Versión española de José Romo Feito).

Lakatos, Imre (1998). Ciencia y pseudociencia. Martin Curd - J.A. Cover, Philosophy.

Lenk, Hans (1988). Entre la epistemología y la ciencia social. Barcelona: Alfa.

Losse, John (1976). Introducción histórica a la filosofía de la ciencia. Madrid: Alianza Editorial. (Versión española de A. Montesinos).

Morin, E. (1983). El método (I). La vida de la vida, Madrid: Cátedra.

Morin, E. (1998). Los siete saberes para la educación del futuro. Madrid.

Morrisey, George L. (1996). Planeación a largo plazo. Creando su propia estrategia. México: Prentice-Hall Hispanoamericana.

Mosterín, Jesús (1994). Filosofía de la cultura. Madrid: Alianza Universidad.

Obando, Octavio y Solís, Luis (2004). Filosofía: inicio y camino. Serie Ciencias Sociales. Lima: Fondo Editorial del Pedagógico San Marcos.

Popper, Karl (1982). La lógica de la investigación científica. Editorial Tecnos, 6. ${ }^{a}$ ed.

Popper, Karl (1997). El mito del marco común. En defensa de la ciencia y la nacionalidad. Barcelona: Paidós Ibérica (versión española de Mario Esquenazi).

Wahl, Jean (1964). Introducción a la filosofía. México: Fondo de Cultura Económica.

Williams, Alan (1965). Finanzas públicas y políticas presupuestarias. Buenos Aires: Amorrortu editores. 